\title{
Variability of low-luminosity AGNs: a simultaneous X-ray/UV look with Swift
}

\author{
P. Romano ${ }^{*},{ }^{a}$ E. Pian, ${ }^{b}$ D. Maoz, ${ }^{c d}$ A. Cucchiara, ${ }^{e}$ C. Pagani, ${ }^{e}$ V. La Parola, ${ }^{a}$ \\ aINAF, Istituto di Astrofisica Spaziale e Fisica Cosmica, \\ Via U. La Malfa 153, I-90146 Palermo, Italy \\ ${ }^{b}$ INAF-Osservatorio Astronomico di Trieste, Via G. Tiepolo 11, Trieste, I-34143, Italy \\ via E. Bianchi 46, I-23807 Merate, Italy \\ ${ }^{c}$ School of Physics and Astronomy, Tel-Aviv University, Tel-Aviv 69978, Israel \\ ${ }^{d}$ INAF-Osservatorio Astrofisico di Arcetri, Largo E. Fermi 5, Firenze, I-50125, Italy \\ ${ }^{e}$ Department of Astronomy \& Astrophysics, Pennsylvania State University, 525 Davey \\ Laboratory, University Park, PA 16802 \\ E-mail: romanodifc.inaf.it
}

\begin{abstract}
We present the results of an investigation of the X-ray and UV properties of four LINERs observed with Swift, aimed at constructing good S/N and strictly simultaneous UV-X-ray SEDs. In the current paradigm, LINER emission is dominated by geometrically thick, radiatively inefficient radiation flows (RIAFs) as opposed to radiatively efficient, geometrically thin accretion disks thought to power higher luminosity AGNs (Seyferts and QSOs). However, some recent studies have found more similarities than differences between the SEDs of LINERs and those of more luminous AGNs, suggesting that LINERs are powered by the same mechanisms active in higher luminosity AGNs. Our new observations allow us to test this idea. In particular, XRT affords long and sensitive monitoring of the X-ray emission. We detect significant variability in M81 and, for the first time, in NGC 3998. The maximum amplitude variations over time scales of some hours are $30 \%$ in both M81 and NGC 3998. NGC 3998 exhibits a variation of the same amplitude on a time scale of 9 days. M81 varies significantly over 2 years, with a maximum change of a factor 2 in 6 months. The X-ray variability detected in 2 of our sources, and in particular in NGC 3998, puts into question the interpretation of their powering mechanism as an inefficient accretion flow, because one of the characteristics of this model is the lack of variability. The identification of NGC 3998 with a low power AGN appears more viable.
\end{abstract}

7th INTEGRAL Workshop

September 8-11 2008

Copenhagen, Denmark

\footnotetext{
${ }^{*}$ Speaker.
} 


\section{Introduction}

Low-luminosity AGNs are so common that virtually all massive galaxies may host some weak activity unrelated to stellar processes. Therefore, in order to understand super massive black hole activity in the local Universe and its evolution, it is necessary to study the demographics and physics of these objects. In particular, Low-Ionization Nuclear Emission Line Regions (LINERs) are lowluminosity active nuclei (AGNs) defined on the basis of their optical spectral line ratios [5, 7], of which several subclasses may be defined according to their properties [2]. The main open question is the origin of their optical spectrum and multi-wavelength emission in general: which fraction of the power source is non stellar and, if it is due to accretion, what is the accretion regime and the radiation conversion efficiency? It has been claimed that the lack of X-ray variability, the non detection of broad $\mathrm{Fe} \mathrm{K} \alpha$ lines down to stringent limits [15], and the weakness or absence of the characteristic "big blue bump" in their optical/near-ultra-violet (UV) spectra, traditionally observed in Seyferts [16, 3], indicate that their engines may be intrinsically different from those of the more luminous AGNs and could consist of radiatively inefficient accretion flows.

However, X-ray observations of these sources have been so far sparse and not sensitive enough to detect variability. Furthermore, ultra-violet (UV) monitoring with the Hubble Space Telescope (HST) Advanced Camera for Surveys (ACS) of a sample of 17 LINERs has revealed the presence of bright and variable UV nuclei [12]. [11], by coupling these UV measurements with non simultaneous X-ray measurements with ASCA, Chandra, and XMM-Newton, has demonstrated that the UV-to-X-ray flux ratios in LINERs are similar to those of Seyferts (although the former sources are much less luminous than the latter). Therefore, contrary to the common paradigm, LINERs may be qualitatively not very different from Seyferts, but rather may be "scaled-down" analogues of them, with the same emission mechanisms operating in both classes.

Here we concentrate on LINER variability by obtaining, for the first time, extended X-ray and UV datasets for a small sample of LINERs. Swift is well suited to this task because of its flexible and efficient scheduling and because it can provide long, simultaneous and accurate UV and X-ray monitoring.

\section{Sample selection}

Our sample includes the brightest LINERs in the [11] UV sample, M81, NGC 3998, NGC 4203, and NGC 4579, that are all Type I LINERs (i.e., with detected broad $\mathrm{H} \alpha$ components) according to [6]. Data for two of these sources, M81 and NCG 4203 were already available in the Swift archive: M81 (net exposure of $33.7 \mathrm{ks}$ ) has an X-ray Telescope spectrum of excellent quality and even the suggestion of an Fe K $\alpha$ line; NGC 4203 (5.3 ks) has a good XRT spectrum, although slightly under exposed with respect to M81. NGC 3998 and NGC 4579 were observed with Swift for the first time (27.4 and $20.8 \mathrm{ks}$, respectively).

\section{Data analysis}

\subsection{XRT}

The XRT data were processed with standard procedures (XRTPIPELINE v0.11.4), filtering and 
Table 1: XRT spectral fit parameters.

\begin{tabular}{lrrrrrrr}
\hline \hline Name & $\begin{array}{r}\text { Distance } \\
(\mathrm{Mpc})\end{array}$ & $\begin{array}{r}N_{\mathrm{H}}^{\mathrm{G}, \mathrm{a}} \\
\left(\mathrm{cm}^{-2}\right)\end{array}$ & $\begin{array}{r}N_{\mathrm{H}}^{\mathrm{b}} \\
\left(\mathrm{cm}^{-2}\right)\end{array}$ & $\Gamma^{\mathrm{c}}$ & $\chi_{\text {red }}^{2} / \mathrm{dof}$ & $\begin{array}{r}F^{\mathrm{d}} \\
(0.2-1 \mathrm{keV})\end{array}$ & $F^{\mathrm{d}}$ \\
\hline M81 & 3.6 & 5.55 & $10.45_{-0.89}^{+0.93}$ & $2.04_{-0.04}^{+0.04}$ & $0.966 / 290$ & $11.2_{-0.1}^{+0.1}$ & $14.9_{-0.4}^{+0.4}$ \\
NGC 3998 & 13.1 & 1.01 & $6.85_{-1.20}^{+1.26}$ & $1.95_{-0.06}^{+0.06}$ & $0.948 / 188$ & $6.2_{-0.8}^{+0.1}$ & $9.8_{-0.4}^{+0.5}$ \\
NGC 4203 & 15.1 & 1.11 & $2.74_{-2.74}^{+4.51}$ & $1.81_{-0.21}^{+0.24}$ & $0.699 / 16$ & $1.4_{-0.4}^{+0.2}$ & $2.8_{-0.4}^{+0.6}$ \\
NGC 4579 & 21 & 2.97 & $7.01_{-1.34}^{+1.43}$ & $1.92_{-0.07}^{+0.07}$ & $0.972 / 145$ & $4.1_{-0.1}^{+0.1}$ & $6.8_{-0.4}^{+0.4}$ \\
\hline
\end{tabular}

${ }^{\text {a }}$ Hydrogen column densities derived from [0] in units of $10^{20} \mathrm{~cm}^{-2}$ and consistent with the $A_{B}$ extinction values reported in [1], using a typical Milky Way gas-to-dust ratio, $5 \times 10^{21} \mathrm{~cm}^{-2}$ mag $^{-1}$.

${ }^{\mathrm{b}}$ Hydrogen column densities from the XRT spectral fits in units of $10^{20} \mathrm{~cm}^{-2}$.

${ }^{\mathrm{c}}$ Photon index, $f_{E} \propto E^{-\Gamma}$.

${ }^{\mathrm{d}}$ Unabsorbed flux in units of $10^{-12} \mathrm{erg} \mathrm{cm}^{-2} \mathrm{~s}^{-1}$.

screening criteria by using FTOOLS in the HEASOFT package (v.6.3.1). We extracted spectra for each XRT observation, as well as for the cumulative observing campaigns. We also extracted light curves in the $0.2-10 \mathrm{keV}$ (total), $0.2-1 \mathrm{keV}$ (soft, S) and 1-10 keV (hard, H) bands. The host galaxy contribution in the XRT extraction region was evaluated using archival Chandra images of these LINERs obtained with ACIS-S with the longest exposure and was found to range from $6 \%$ (NGC 3998) to $16 \%$ (NGC 4203), and was subsequently ignored. We fit the mean XRT spectrum for each object with a simple absorbed power-law model with free absorption and photon index (see Table 1). The spectra show no significant absorption features superimposed on the power-law continuum. Although the uncertainties on the fitted Hydrogen column densities (Table 1. Col. 4) are not small, all sources show significant evidence for an absorption in excess of the Galactic one (also reported in Table 1).

\subsection{UVOT}

The Swift UltraViolet-Optical Telescope observed the four targets with the filters $u$ (3465 $\AA$ ) $u v w 1(2600 \AA), u v m 2(2246 \AA), u v w 2(1928 \AA)$. The filter choice was driven by the objective of maximizing the nuclear signal, which is dominant in the UV, while minimizing the emission from the bulges of the galaxies. After verifying that the UVOT counts show no significant variability, we have coadded the images for each object. The data analysis was performed using the UVOTSOURCE task included in the latest HEASOFT software.

Using archival HST images of these LINERs at $2500 \AA$ and $3300 \AA$ obtained with the High Resolution Channel (HRC) of the HST ACS [12], we evaluated the host galaxy contribution for each object in a 2 arcsec-radius circular area centered on the nucleus. The resulting magnitudes were converted into fluxes using the latest in-flight flux calibration factors and zero-points [14]. The host galaxy contribution is dominant in the $u$ band (generally comparable to the total flux observed by UVOT; sometimes it exceeds it by 20 to $50 \%$ ) and it decreases almost steadily toward 
the shorter wavelengths. It is minimal in the $u v w 2$ filter ( $1928 \AA$ ) where it contributes at most $60 \%$ of the observed flux (NGC 3998).

\subsection{Results}

Significant X-ray variability is detected in NGC 3998 for the first time. The variations are about $30 \%$ on both intraday and interday time scales. In Fig. 1]c is reported the light curve of one of the 2 Swift pointings we have made and in Fig. $1 \mathrm{~d}$ are reported the average fluxes of the 2 pointings, in soft and hard X-rays. This is unprecedented for this source, that has always been reported not to be variable in the X-rays [13, 15]. M81 varies with a maximum amplitude of $30 \%$ on intra-day time-scales. In Fig. 1]a we report the light curve of one of our 5 observations, in which both the soft and hard X-rays are seen to vary in a correlated way, although the significance of the soft X-ray variation is limited. The month time scale variability has a larger amplitude, up to a factor 2 in 6 months in both soft and hard X-rays, and good correlation between the 2 bands (Fig. 1b). This is similar to previous findings; indeed, earlier X-ray observations of M81 had reported variations of even a factor 4 [10]. Variability on a minute time scale is seen of up to 30-40\%, but it occurs on low states of the sources, and therefore we consider it highly dubious.

NGC 4579 and NGC 4203 (Fig. 17, f), on the other hand, exhibit no significant variability on any time scale. One reason may be that our monitoring was carried out for too short a baseline, over which there was no chance to detect variations, that occur stochastically.

After host galaxy subtraction, we do not detect significant optical/UV variability in any of our sources.

\subsection{Conclusions}

The variability we find is not dissimilar from that of Seyferts. While this does not prove that LINERs have (weak) AGNs at their centers as opposed to advection dominated accretion flows (ADAF), it puts the issue back into question. In particular, we detect significant variability in an object (NGC 3998) that had been considered a very good example of an ADAF, based on the lack of variability [13, 15].

In summary, we suggest that, if monitored with sufficient accuracy, long time and on a wide band, some LINERs can vary, albeit with modest amplitude. This is not inconsistent with the variations of Seyferts. A real test of the emission model calls for a multiwavelength approach. Our UV observations are of limited help, because they are affected by the host galaxy and are therefore not sufficiently accurate to study UV variability. A compelling test would consist in strictly simultaneous XRT and radio observations, possibly accompanied by sensitive HST observations in the UV. In a forthcoming paper we will present the results of the analysis of the simultaneous X-ray and UV spectral energy distributions [17]. 


\section{Acknowledgments}

We thank N. Gehrels for approving this set of ToOs and the Swift team, in particular the duty scientists and science planners, for making these observations possible. P.R. thanks INAF-IASF Milano, where part of the work was carried out, for their kind hospitality. This research has made use of NASA's Astrophysics Data System Bibliographic Services, as well as the NASA/IPAC Extragalactic Database (NED), which is operated by the Jet Propulsion Laboratory, California Institute of Technology, under contract with the National Aeronautics and Space Administration.

\section{References}

[1] Cappi, M., Panessa, F., Bassani, L., et al., 2006, A\&A, 446, 459

[2] Chiaberge M., Capetti A., Macchetto F.D., 2005, ApJ, 625, 716

[3] Chiaberge M., Gilli R., Macchetto F.D., Sparks W.B., 2006, ApJ, 651, 728

[4] Elvis, M., Wilkes, B.J., McDowell, et al., 1994, ApJS, 95, 1

[5] Heckman T.M. 1980, A\&A, 87, 152

[6] Ho L.C., Filippenko A.V., Sargent W.L.W., et al. 1997, ApJS, 112, 315

[7] Ho L.C. 2008, AARA, in press [arXiv:0803.2268]

[8] Ho, L.C., Feigelson, E.D., Townsley, L.K., et al., 2001, ApJ, 549, L51

[9] Kalberla, P.M.W., Burton, W.B., Hartmann, et al., 2005, A\&A, 440, 775

[10] La Parola, V., Fabbiano, G., Elvis, M., et al. 2004, ApJ, 601, 831

[11] Maoz D., 2007, MNRAS, 377, 1696

[12] Maoz, D., Nagar, N. M., Falcke, H., et al. 2005, ApJ, 625, 699

[13] Pellegrini S., Cappi M., Bassani L., et al. 2000b, A\&A, 360, 878

[14] Poole T.S., Breeveld A.A., Page M.J., et al. 2008, MNRAS, 383, 627

[15] Ptak, A., Terashima, Y., Ho, L.C., et al. 2004, ApJ, 606, 173

[16] Quataert E., Di Matteo T., Narayan R., et al. 1999, ApJ, 525, L89

[17] Romano, P., Pian, E., Maoz, D., et al., 2009, MNRAS, in preparation

[18] Terashima, Y., Iyomoto, N., Ho, L.C., et al. 2002, ApJS, 139, 1 

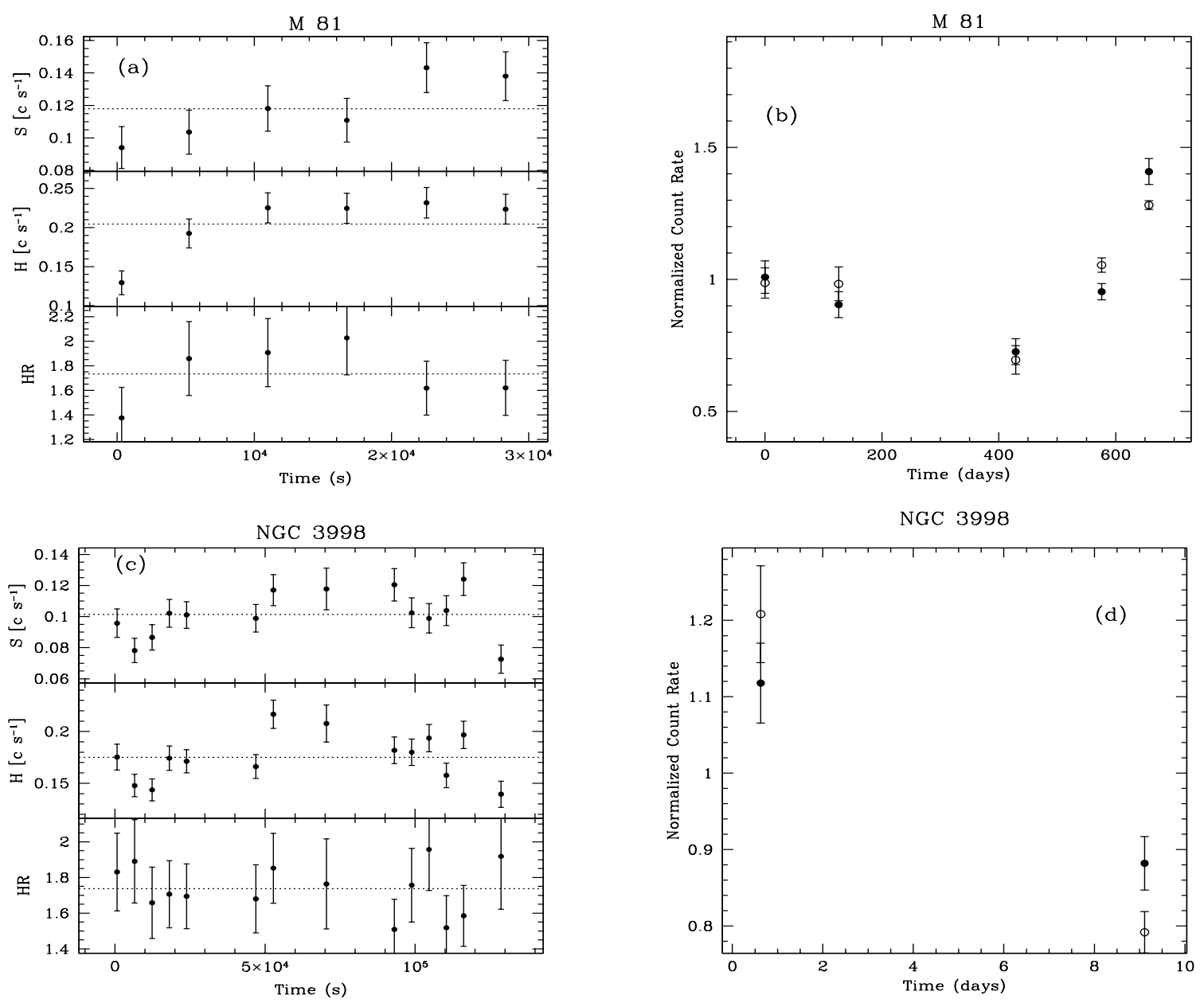

Figure 1: Swift/XRT background-subtracted light curves (count rate in counts $\mathrm{s}^{-1}$ ) and hardness ratios (i.e., 1-10 keV to $0.2-1 \mathrm{keV}$ flux ratios): (a) $0.2-1 \mathrm{keV}$ (top), $1-10 \mathrm{keV}$ (middle) light curves and hardness ratio curves (bottom) of M81 starting on 2006 June 24.004 UT. The binning time interval corresponds to the orbit duration. (b) 0.2-1 keV (filled circles) and 1-10 keV (open circles) light curves of M81 between 2005 and 2007. Each curve is normalized to its average $\left(0.16\right.$ and 0.28 counts $\mathrm{s}^{-1}$ for the $0.2-1 \mathrm{keV}$ and $1-10 \mathrm{keV}$ curves, respectively). Each point is the average of the flux measured during each of the 5 pointings in that given band. The time origin corresponds to 2005 Apr 21.0 UT. (c) $0.2-1 \mathrm{keV}$ (top), $1-10 \mathrm{keV}$ (middle) light curves and hardness ratio curves (bottom) of NGC 3998 starting on 2007 April 29.11 UT. The binning time interval corresponds to the orbit duration. (d) $0.2-1 \mathrm{keV}$ (filled circles) and $1-10 \mathrm{keV}$ (open circles) light curves of NGC 3998 averaged over each of the two pointings in April 2007. Each curve is normalized to its average ( 0.11 and 0.21 counts $\mathrm{s}^{-1}$ for the $0.2-1 \mathrm{keV}$ and $1-10 \mathrm{keV}$ curves, respectively). The time origin corresponds to 2007 Apr 20.0 UT. 

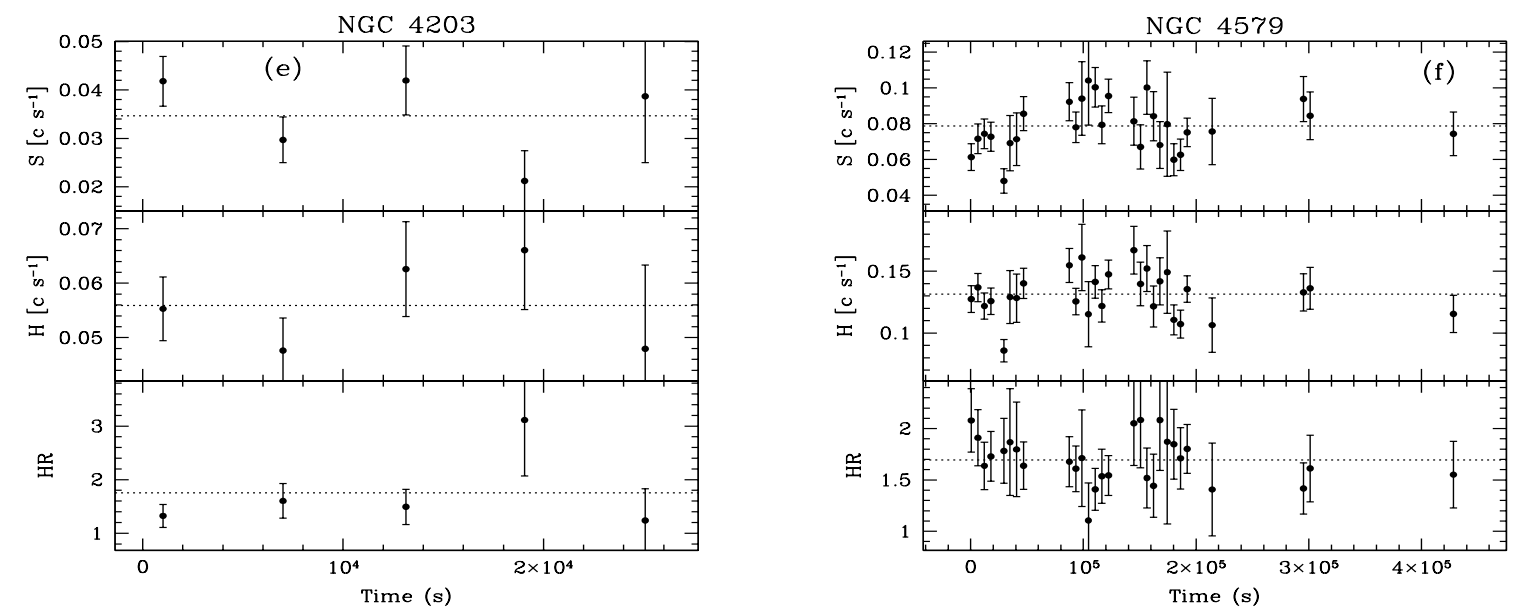

Figure 1: (e) 0.2-1 keV (top), 1-10 keV (middle) light curves and hardness ratio curves (bottom) of NGC 4203 starting on 2005 December 25.004 UT. The binning time interval is the orbit duration. $(f) 0.2-1$ $\mathrm{keV}$ (top), 1-10 keV (middle) light curves and hardness ratio curves (bottom) of NGC 4579 starting on 2005 May 15.04 UT. The binning time interval is the orbit duration. 